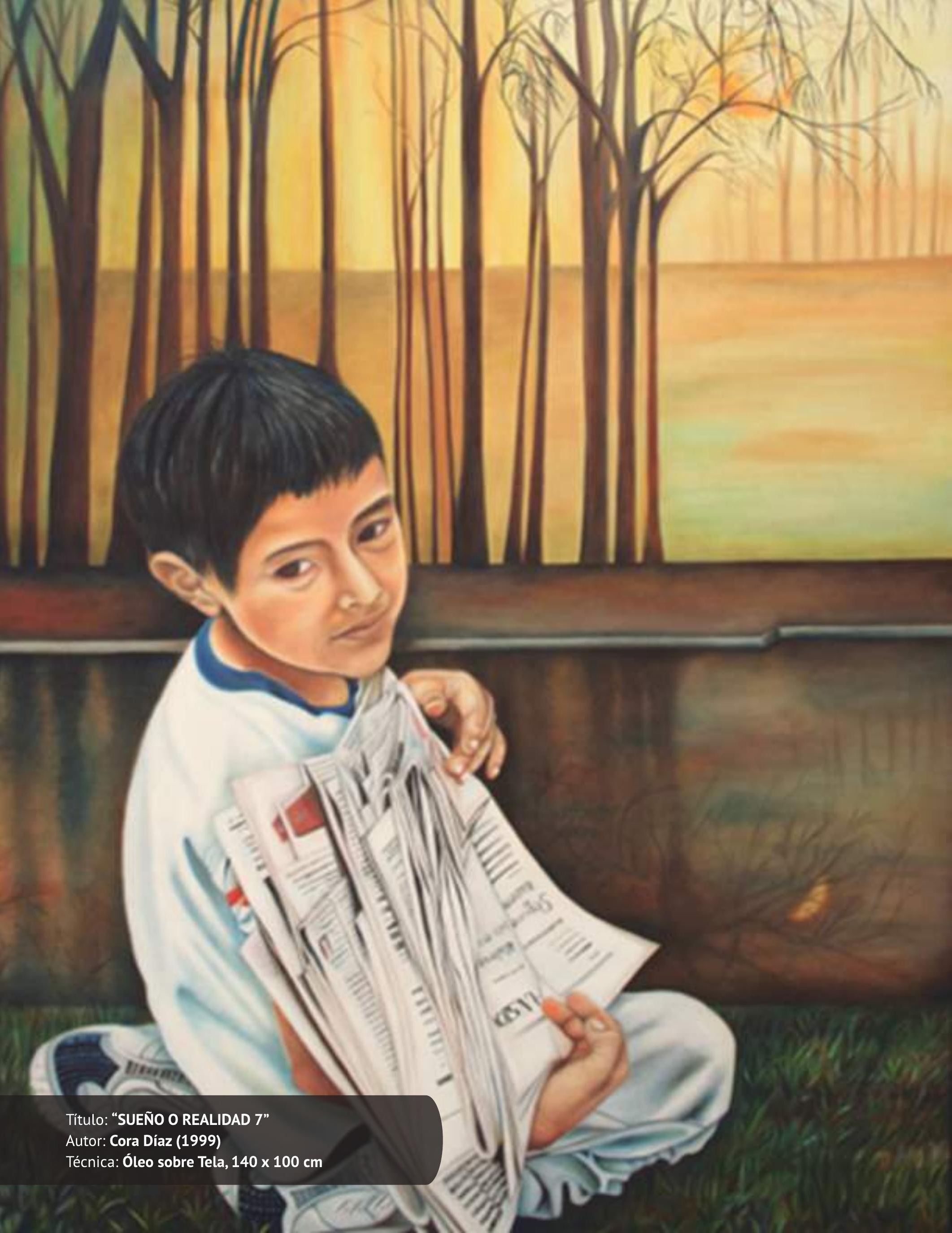




\section{EFECTOS DE LAS ANFETAMINAS EN EL SISTEMA NERVIOSO}

EFFECTS OF AMPHETAMINES

ON THE NERVOUS SYSTEM

\section{RAFAEL VILLARREAL GUAJARDO LUIS ANTONIO CÓRDOVA RIVERA}

\section{RESUMEN}

Debido al incremento exponencial del consumo de anfetaminas en nuestro estado, ha surgido un problema social que repercute directamente en el entorno en el cual puede ser cualquier persona y, por la situación actual, el adolescente es uno de los individuos más susceptibles de caer en la experimentación y adicción a esta sustancia. Los efectos en la sinapsis del consumidor repercuten en un deterioro orgánico, que en un proceso de adicción regularmente son irreversibles. A través del presente trabajo se pretende incidir de manera directa en la conciencia de los adolescentes, dándoles a conocer los daños que se causan al consumir estas sustancias, previendo la no proliferación de dichas adicciones.

PALABRAS CLAVE: ANFETAMINAS, SINAPSIS, CRISTAL, ADICCIÓN, DOPAMINA, CONSECUENCIAS.

\section{ABSTRACT}

Due to the exponential increase in the consumption of amphetamines in our state, a social problem has arisen that has a direct impact on the user's environment which can be any person and, due to the current situation, the adolescent is one of the most susceptible individuals to fall into experimentation and addiction to this substance; the effects on the consumer's synapse have an organic deterioration, which in an addiction process is regularly irreversible. Through this work, it is intended to directly influence adolescents' awareness, informing them of the damages caused by consuming these substances, providing for the non-proliferation of these addictions.

KEYWORDS: AMPHETAMINES, SYNAPSES, CRYSTAL, ADDICTION, DOPAMINE, CONSEQUENCES. 
$\mathrm{E}$ I sistema nervioso, junto con el endocrino, regula todas las funciones de nuestro cuerpo, tiene relación con todo lo que hacemos, sentimos y pensamos, y nos permite conectarnos con el medio ambiente externo e interno (por diferentes procesos que se llevan a cabo dentro del cuerpo). En este caso nos centramos en la funcionalidad de las neuronas (las células que transmiten la información en dicho sistema) y la sinapsis (el espacio a través del que se comunican las neuronas).

Las metanfetaminas son medicamentos que fueron creados para tratar la fatiga, mejorar la resistencia y estado de alerta, tratar la obesidad, la narcolepsia, que es la excesiva somnolencia diurna, (Peraita Adrados, del Río Villegas y Vela Bueno, 2015) y la depresión. Actualmente se han estado utilizando para el tratamiento de trastorno por déficit de atención y su uso puede generar graves consecuencias en la sinapsis de las vías dopaminérgicas (la dopamina es una sustancia neurotransmisora relacionada con el placer a nivel cerebral y con los movimientos musculares).

\section{PROBLEMÁTICA}

De acuerdo al Sistema de Vigilancia Epidemiológica de las Adicciones de Nuevo León (SISVEA, 2019), el consumo de metanfetaminas se ha ido incrementando desde el año 2014 en nuestro estado, por esta razón se está convirtiendo en un problema social que abarca desde la adolescencia afectando la etapa de desarrollo y formación del adulto.

En el informe 2018 de la situación de consumo de drogas en México y su atención integral, la directora

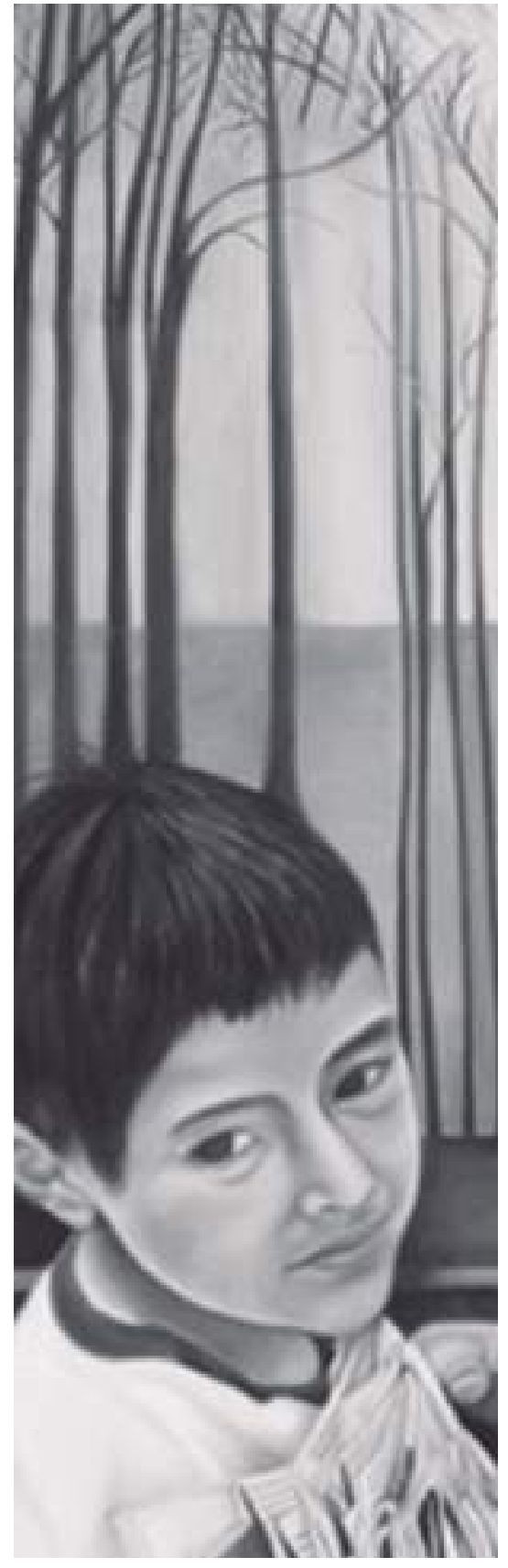

general de Atención y Tratamiento de la Comisión Nacional Contra las Adicciones (CONADIC) aseguró que los estimulantes de la familia de anfetaminas se utilizaron en mayor cantidad que el cannabis, desplazando a esta hierba al 2do lugar en consumo (Secretaría de Salud, 2019).

Las anfetaminas están causando una problemática de alerta a nivel local, estatal y nacional debido a que pueden originar adicción desde la primera vez que se consumen, también por la excitación que se genera al estar bajo los influjos de esta droga se puede generar sexo de riesgo 0 episodios violentos.

La pregunta de investigación del presente trabajo es la siguiente: ¿Cuáles son los efectos de las metanfetaminas en la sinapsis dopaminérgica? Los objetivos de esta investigación se mencionan a continuación:

1. Identificar cuáles son los efectos de las metanfetaminas en la sinapsis dopaminérgica.

2. Identificar cuáles son las consecuencias macroscópicas de los efectos del uso de metanfetaminas en el sistema nervioso.

La metodología utilizada es de investigación documental. problema sociosanitario. Las principales complicaciones son la patología neurovascular, la infección, los trastornos tóxicometabólicos y la atrofia cerebral 
A continuación se menciona la hipótesis planteada. Los efectos de las metanfetaminas en las vías dopaminérgicas son:

- La excesiva expulsión en la dopamina.

- La destrucción de receptores dopaminérgicos por exceso de dopamina en el espacio sináptico.

Las drogas vienen siendo un gran problema sociosanitario. Las principales complicaciones son la patología neurovascular, la infección, los trastornos tóxico-metabólicos y la atrofia cerebral. La sintomatología inespecífica y la negación del consumo hacen que la implicación del radiólogo pueda resultar fundamental en la atención de estos pacientes. La neuroimagen permite detectar alteraciones precoces y plantear el diagnóstico etiológico ante patrones de afectación específicos (Montoya Filardi y Mazón, 2017).

Las metanfetaminas se consiguen de forma regular mediante eventos en donde se reúnen los adolescentes (en las llamadas reu's o revento-

\section{Uno de los neurotransmisores más importantes es la dopamina la cual está involucrada en varios desórdenes neurológicos y mentales (Corominas, M; Roncero, C; Bruguera E. \& Casas, M., 2007)}

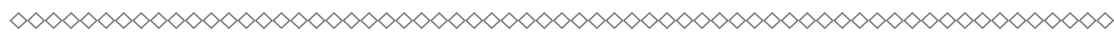

nes), así como en eventos masivos que duran toda la noche y donde se pueden obtener de forma sencilla las bebidas alcohólicas, todo esto con la intención de maximizar el éxtasis de la fiesta, lo cual pone en riesgo sus decisiones (Secretaría de Salud, CONADIC, 2017).

Su fórmula química es un anillo bencénico, sustituida por dos radicales (un amino y un metil). Estas sustancias son muy parecidas químicamente a la adrenalina. Sin embargo, desde el punto de vista farmacológico, a medida que un compuesto de estructura química semejante a la adrenalina se va apartando de ella para aproximarse a las anfetaminas, éste va aumentando su actividad estimulante del SNC y disminuyendo su actividad sobre la periferia del organismo (Robledo, 2008).

Uno de los neurotransmisores (sustancias mediante las cuales se comunican las neuronas) más importantes es la dopamina (responsable de provocar placer), la cual está involucrada en varios desórdenes neurológicos y mentales. Se deriva de la fenilalanina y se sintetiza en las neuronas de la sustancia negra del mesencéfalo (estructura cerebral relacionada con la

TABLA 1. Características, Efectos y Repercusiones de las Anfetaminas (Secretaría de Salud, Conadic, 2019)

\begin{tabular}{|c|c|c|c|}
\hline & CARACTERÍSTICAS & EFECTOS & REPERCUSIONES \\
\hline Nombres & $\begin{array}{c}\text { Speed, Anfetas, } \\
\text { Elevadores, Tacha, Cristal, } \\
\text { Éxtasis, Cruz Blanca. }\end{array}$ & \multirow{3}{*}{$\begin{array}{l}\text { - Excitación } \\
\text { - Temblor de manos } \\
\text { - Sudoración abundante } \\
\text { - Insomnio } \\
\text { - Disminucion de apetito } \\
\text { - Nerviosismo } \\
\text { - Depresión } \\
\text { En el bajón se puede } \\
\text { experimentar paranoia } \\
\text { y agresión. }\end{array}$} & \multirow{3}{*}{$\begin{array}{l}\text { - Irritabilidad } \\
\text { - Agresividad } \\
\text { - Fiebre } \\
\text { - Boca y nariz resecas } \\
\text { - Convulsiones } \\
\text { - Alucinaciones } \\
\text { - Delirios de persecución } \\
\text { - Desórdenes mentales }\end{array}$} \\
\hline Presentación & $\begin{array}{c}\text { Tabletas o Cápsulas } \\
\text { (tamaño, color y forma } \\
\text { diversas). }\end{array}$ & & \\
\hline Consumo & $\begin{array}{c}\text { Polvo, Trozo inhalados, } \\
\text { tragados, inyectados o } \\
\text { fumados medante una Pipa. }\end{array}$ & & \\
\hline
\end{tabular}


coordinación muscular) (Corominas, M; Roncero, C; Bruguera E. \& Casas, M., 2007).

El consumo continuado de metanfetamina causa cambios en el sistema de dopamina del cerebro asociados con una menor coordinación y con la disminución del aprendizaje verbal. En estudios de personas que consumieron metanfetamina durante mucho tiempo se observó que los cambios profundos también afectaron las áreas del cerebro que están relacionadas con la emoción y la memoria. Esto puede explicar muchos de los problemas emocionales y cognitivos que se ven en quienes consumen metanfetamina.

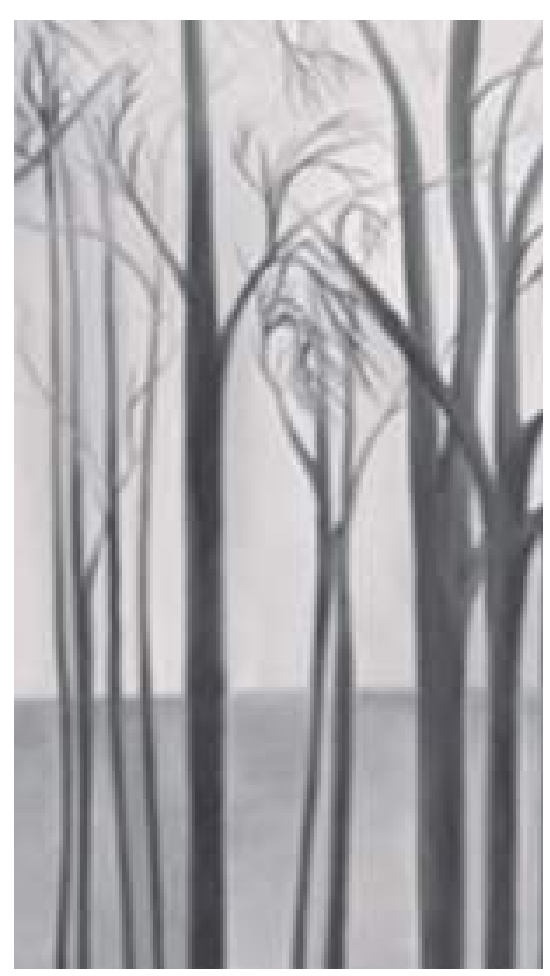

FIGURA 1. Fórmula Química de la Anfetamina y Metanfetamina
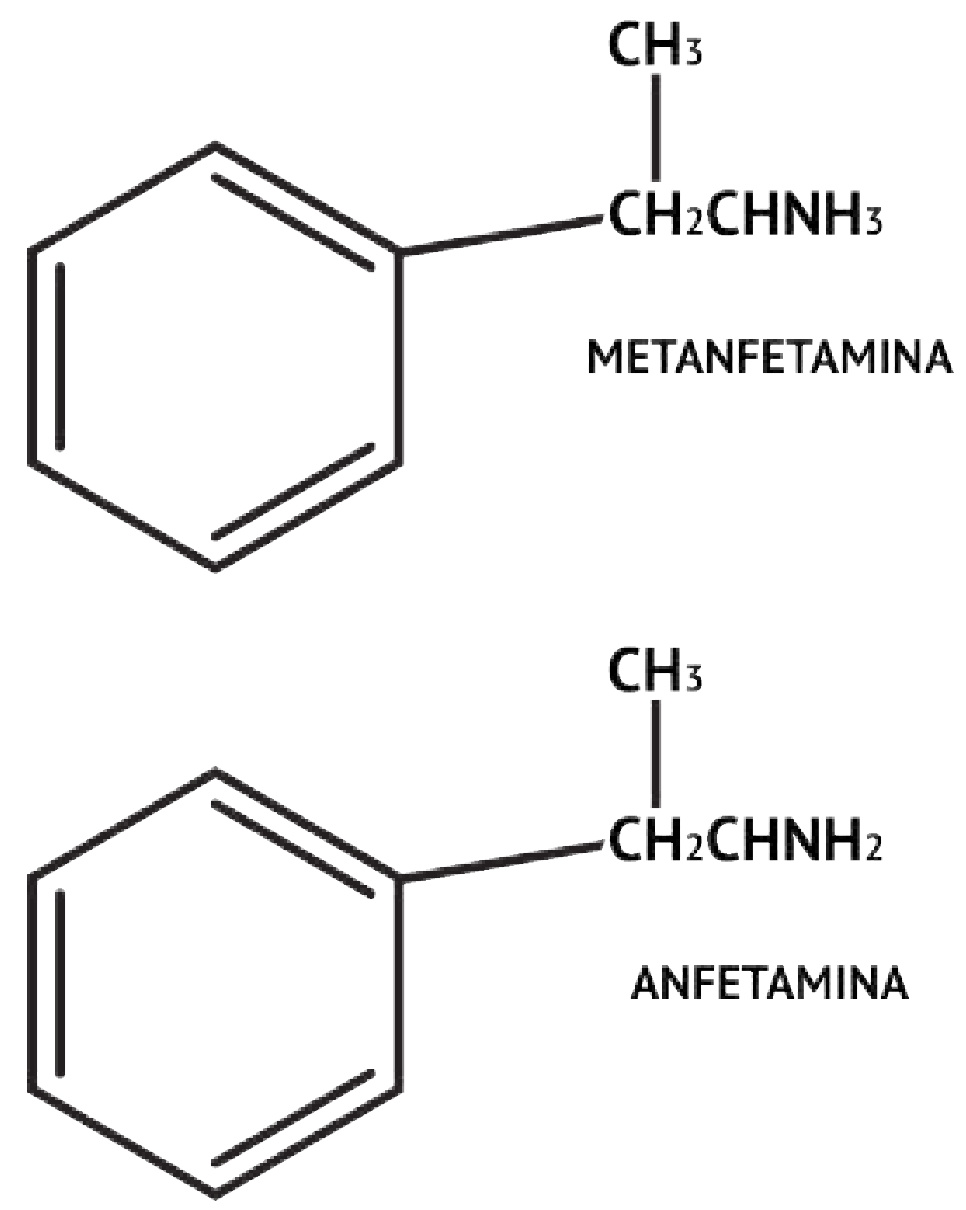

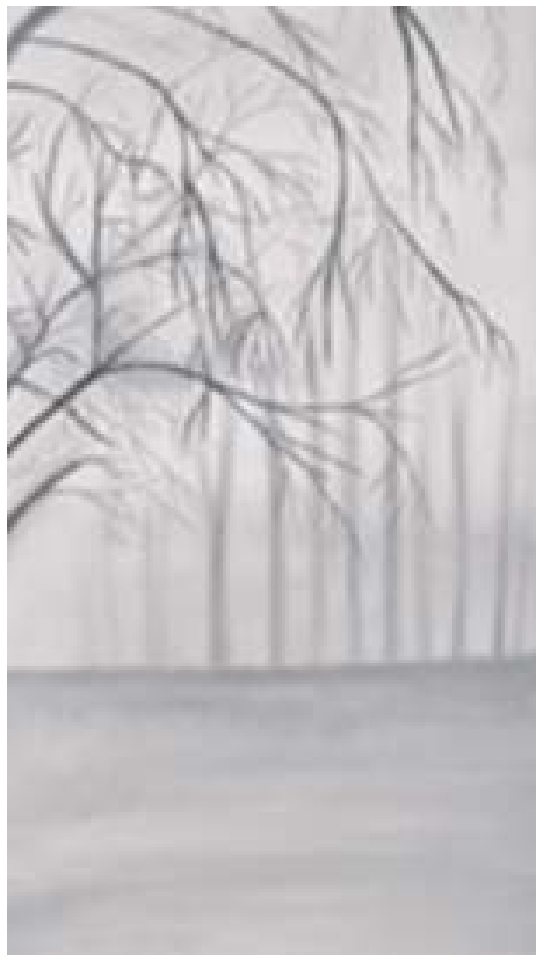

En un estudio se encontró que las anfetaminas tienen un efecto neurotóxico en las neuronas dopaminérgicas postsinápticas, debido a la excitación para secretar dopamina en grandes cantidades, lo cual genera inflamación, estrés oxidativo, hipertermia y disfunción mitocondrial, lo cual se ha presentado en modelos animales (siendo aparentemente similar al humano) en donde se ha investigado al respecto.

En este sentido, Robledo (2008) comenta que la neurotoxicidad producida por las anfetaminas normalmente se refiere a alteraciones persistentes a nivel neuroquímico y neuronal, incluyendo lesiones en los axones y terminaciones que contienen monoaminas.

Entre los efectos potencialmente mortales destacan la hemorragia en el sistema nervioso, la hemorragia intracraneal, el infarto cerebral y la trombosis del seno venoso cerebral. 


\section{CONCLUSIÓN}

La hipótesis se acepta, pues hay evidencia de daño en las vías dopaminérgicas. Al haber exceso de neurotransmisor en el espacio sináptico existe una sobrecarga de trabajo en los receptores de dicha sustancia, lo cual lleva a la perdida de receptores dopaminérgicos como un mecanismo de defensa del cuerpo, provocando la necesidad de mayor cantidad de droga para lograr los efectos deseados al momento de administrarse la sustancia psicótropa.

\section{PROPUESTA}

En conjunto con los Centros de Atención Primaria en Adicciones (CAPA) del estado de Nuevo León, se aplicará un tamizaje de factores de riesgo que identifiquen situaciones que puedan orillar a los adolescentes al consumo de drogas, al procesar los datos se reconocerán las personas en peligro y se enfocará en ellos la estrategia de sensibilización. Además se buscará coadyuvar en el trabajo del Comité Municipal Contra las Adicciones del municipio de Guadalupe, Nuevo León.

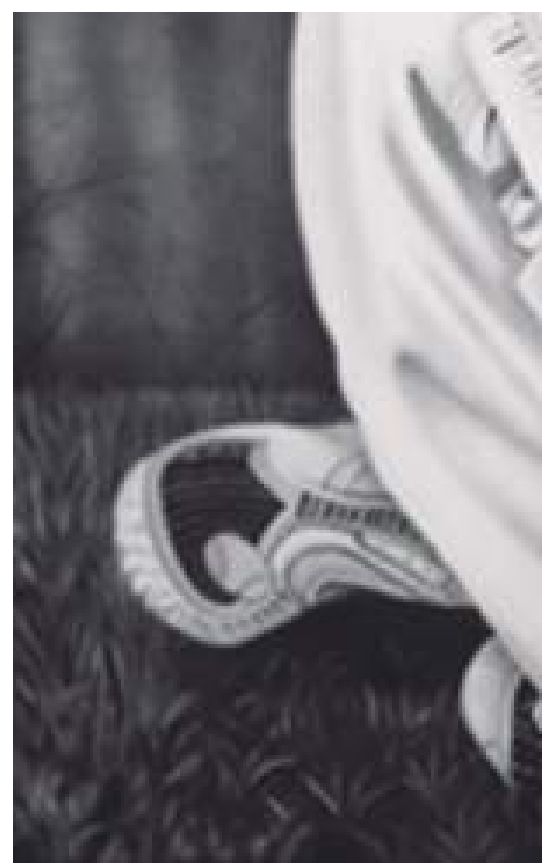

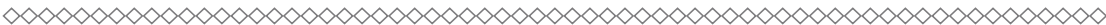

Robledo comenta que la neurotoxicidad producida por las anfetaminas normalmente se refiere a alteraciones persistentes a nivel neuroquímico y neuronal, incluyendo lesiones en los axones y terminaciones que contienen monoaminas

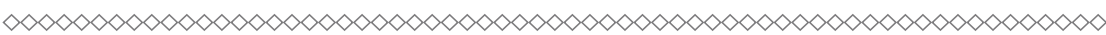
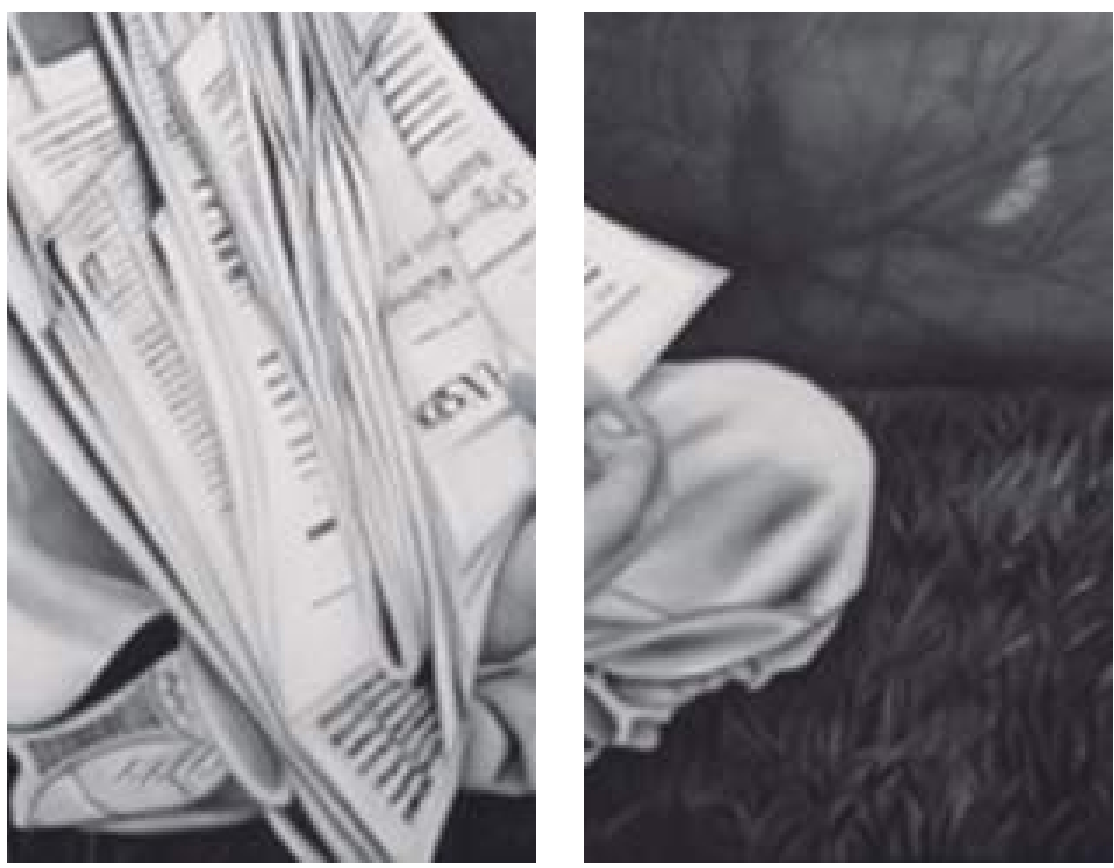

Presencia Universitaria 


\section{REFERENCIAS}

Corominas, M, Roncero, C, Bruguera E.y Casas, M. (2007). Sistema dopaminérgico y adicciones. Revista de Neurología, 23-31.

Montoya Filardi, A. y Mazón, M. (2017). El cerebro adicto: imagen de las complicaciones neurológicas por el consumo de drogas. Radiología, 17-31.

Peraita Adrados, R., del Río Villegas, R., y Vela Bueno, A. (2015). Factores ambientales en la etiología de la narcolepsia-cataplejia. Estudio de casos y controles de una serie. Revista de Neurologia, 529-534.

Robledo, P. (2008). Las anfetaminas. Trastornos adictivos, 166-174.

Secretaría de Salud. (2019). Se presenta en México el Informe Mundial de Drogas 2019. México: Gobierno de México.

Secretaría de Salud, CONADIC (2017). CONADIC presenta guías preventivas para niños, niñas, adolescentes, padres y maestros. Obtenido de https://www.gob.mx/salud/conadic/accionesy-programas/conadic-presenta-guias-preventivas-para-ninos-ninas-adolescentes-padres-ymaestros-108566\#documentos.

Secretaría de Salud, CONADIC (2019). Prevención de las adicciones y promoción de conductas saludables. Obtenido de http://www.conadic.salud.gob.mx/pdfs/nueva_vida/nv1e_ prevencion.pdf.

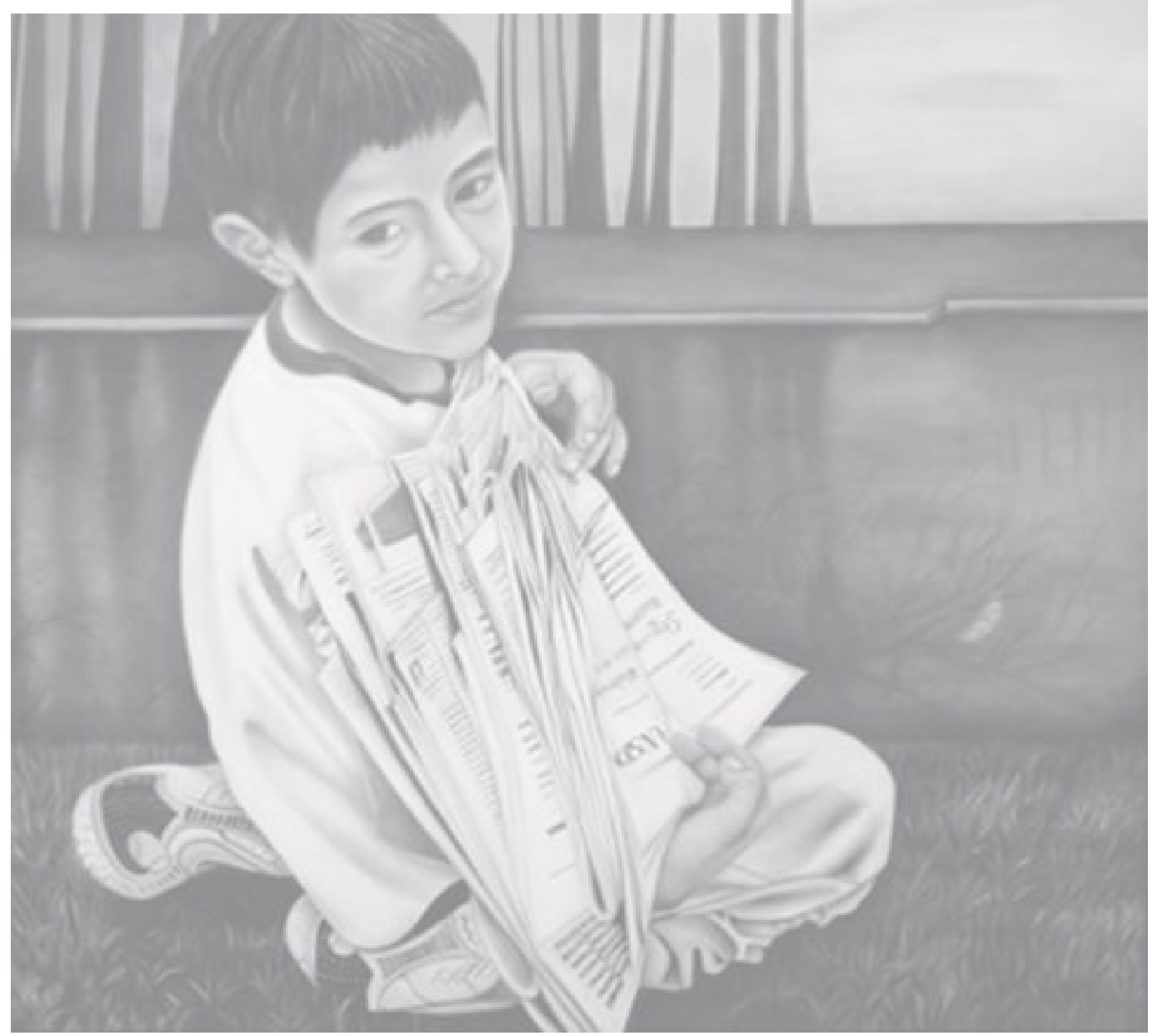




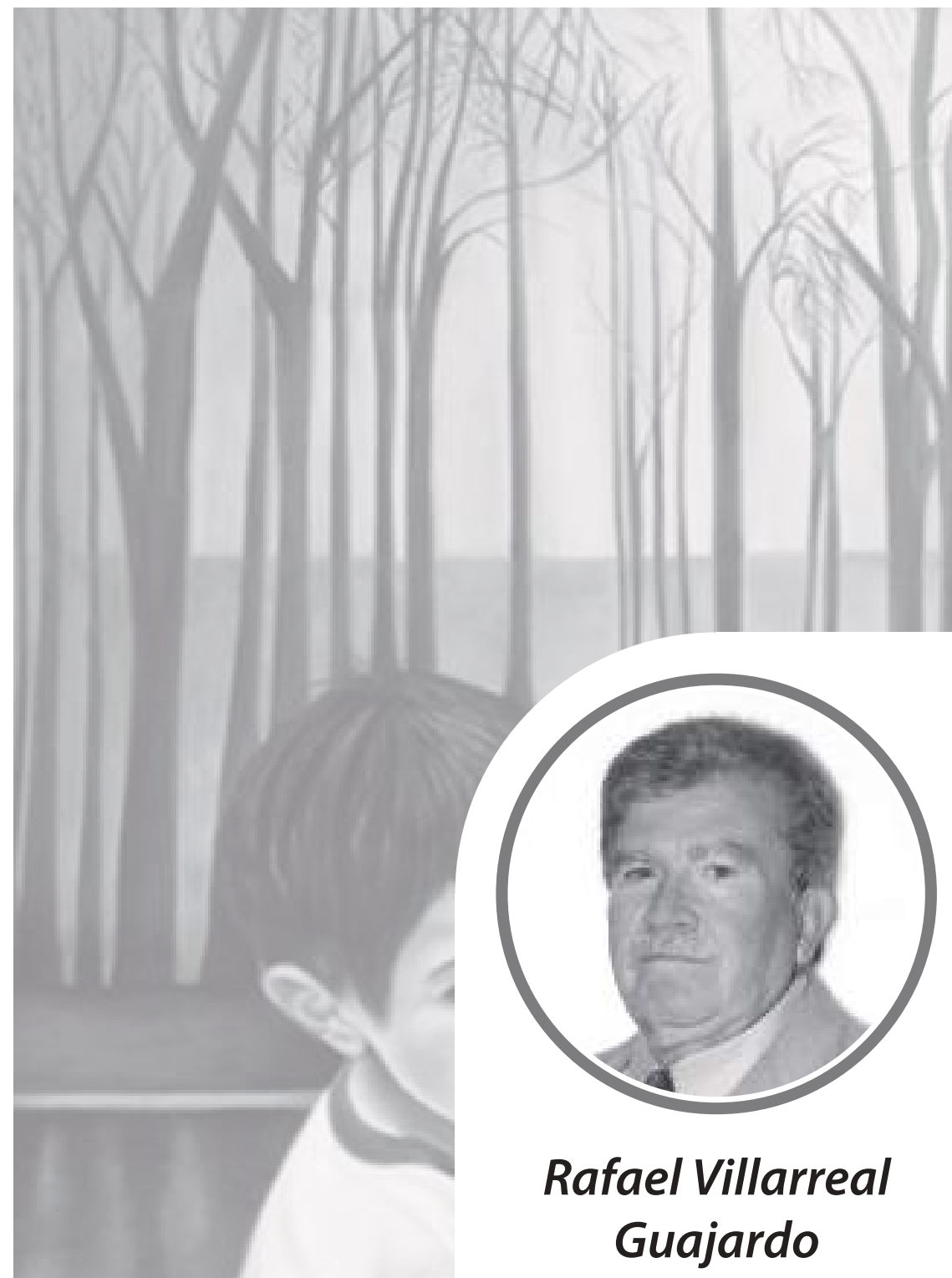

Es egresado de la Facultad de Ciencias Químicas UANL como Ingeniero Químico con Maestría en Ingeniería Industrial y Maestría de Metodología de la Ciencia en el Posgrado de la Facultad de Filosofía y Letras. Perteneció a la Comisión de Honor y Justicia del Consejo Universitario. Desarrolló su profesión en la Compañía Fundidora Monterrey. Actualmente es profesor de tiempo completo y exclusivo titular A, e imparte la cátedra de Química en la Preparatoria 8 de la UANL.

Correo Electrónico: rafavigua@hotmail.com

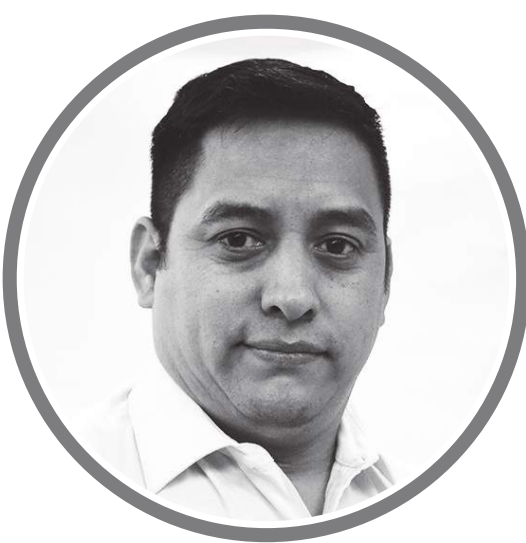

\section{Luis Antonio Córdova Rivera}

Es egresado de la Facultad de Odontología en donde colaboró como instructor de Anatomía Humana y de Operatoria Dental I y IV. Presidente del Colegio de Odontología Infantil de Nuevo León, A.C. en 2013. Es Doctor en Métodos Alternos en Solución de Controversias. Actualmente es profesor de las unidades de aprendizaje Caminos del Conocimiento y Biología II y Jefe del Departamento de Promoción de la Salud en la Preparatoria 8 de la UANL.

\section{Correo Electrónico:}

drluiscprdova@gmail.com

Recibido: 15/09/2019

Aceptado: 15/10/2019 